\title{
Nonlinear Dynamics of Semiconductor Lasers With Mutual Optoelectronic Coupling
}

\author{
Shuo Tang, Raúl Vicente, Margaret C. Chiang, Claudio R. Mirasso, and Jia-Ming Liu
}

\begin{abstract}
Mutually coupled oscillators are currently of great interest because of the important insight they provide into coupled physical, chemical, and biological systems. Two semiconductor lasers with optoelectronic feedback are used as two nonlinear oscillators, and the effect of mutual coupling on these lasers is found to be significant. Depending on the operating conditions, mutual coupling can act as a negative feedback to stabilize the coupled oscillators, or it can increase the complexity of the system inducing a highly complex chaos. A quasi-periodicity and period-doubling bifurcation, or a mix of them, is found in such a system. Although the chaotic waveforms are very complex with broad spectra, a high quality of synchronization between the chaotic waveforms is observed. Such synchronization is achieved because of the effect of mutual coupling and the symmetric design between the two lasers. It is found that the time delay of coupling plays an important role on the dynamics and synchronization in the mutually coupled semiconductor lasers.
\end{abstract}

Index Terms-Chaos, death by delay, mutual coupling, optoelectronic feedback, semiconductor lasers, synchronization.

\section{INTRODUCTION}

$\mathbf{N}$ ONLINEAR dynamics of semiconductor lasers are of great interest because of the important roles these devices play in many significant applications. Chaotic dynamics can be induced in a semiconductor laser by increasing its dimension through a proper external perturbation, such as optical feedback, optical injection, or optoelectronic feedback [1]-[4]. Single-mode semiconductor lasers subject to optoelectronic feedback can exhibit chaotic dynamics under certain operating conditions. Either positive [5] or negative [6] optoelectronic feedback can be applied to a solitary single-mode semiconductor laser to generate a chaotic dynamics. In both cases, the laser follows a quasi-periodicity route to chaos.

While the dynamics of individual semiconductor lasers, either free running or subject to an external perturbation, have been widely investigated, coupled semiconductor lasers have also recently gained attention. Chaos synchronization has been demonstrated in unidirectionally coupled semiconductor laser

Manuscript received January 8, 2004; revised April 21, 2004. This work was supported by the U.S. Army Research Office under Contract DAAG55-98-10269. The work of R.Vicente was supported by the Ministerio de Ciencia Y Technologia (Spain) and Feder (EU) under Project BFM2002-04369 and by the EC under project OCCULT IST-2000-29683. The work of C. R. Mirasso was supported by the Secretaría de Estado de Educación y Universidades, Ministerio de Educación, Cultura y Deporte, Spain, and by Ministerio de Ciencia Y Technologia (Spain) and Feder (EU) under Project BFM2002-04369 and by the EC under project OCCULT IST-2000-29683.

S. Tang, M. C. Chiang, and J.-M. Liu are with the Electrical Engineering Department, University of California, Los Angeles, CA 90095-1594 USA.

R. Vicente and C. R. Mirasso are with the Departament de Fisica, Universitat de les Illes Balears, E-07122 Palma de Mallorca, Spain.

Digital Object Identifier 10.1109/JSTQE.2004.837013 systems [7]-[10] and chaotic optical communication has been widely studied and demonstrated using semiconductor lasers operating in chaotic states [11]-[14]. Mutually coupled semiconductor lasers have received less attention although a wide variety of dynamics have been observed [15]-[17]. In mutually coupled semiconductor lasers, not only that the output of one laser is coupled into the dynamics of the other laser, but also that the time delay introduced by the mutual coupling further increases the dimension of the degree of freedom in the coupled lasers. Consequently, a lot of interesting dynamics have been observed in such mutually coupled semiconductor lasers. For optically coupled devices, localized synchronization of periodic oscillations [15], spontaneous symmetry breaking followed by leader-laggard dynamics, and asymmetric role of the two lasers have been observed [16]. Mutual coupling induces new nonlinear phenomena and significantly changes the dynamics of the lasers. Uncoupled semiconductor lasers are independent nonlinear oscillators. Mutual coupling connects these nonlinear oscillators together. Counterintuitively, mutual coupling has been observed to stabilize and quench the oscillation amplitude of two pulsating oscillators, a phenomenon known as "death by delay." It can also destabilize the nonlinear system to generate highly complex chaos.

In this paper, we study the effect of mutual coupling between two semiconductor lasers with optoelectronic feedback. This paper is organized into six sections, with the first being this introduction. System modeling and numerical results are given in Section II. Beyond this section, our experimental results are presented. Section III covers the phenomenon of "death by delay" while Sections IV and V, respectively, discuss the generation of chaos and the synchronization of such chaos. Lastly, a brief conclusion is given in Section VI.

\section{System Configuration AND Modeling}

It is of great interest to study the effect of mutual coupling on nonlinear oscillators. The configuration of coupled nonlinear dynamical oscillators using two semiconductor lasers is shown in Fig. 1. The two semiconductor lasers, laser diode 1 (LD1) and laser diode 2 (LD2), each with its own optoelectronic feedback loop, are the two mutually coupled elements under study. Within each optoelectronic feedback loop, the laser output is detected and converted into an electronic signal by a photodetector, PD1 or PD2. After amplification the electronic signal is fed back to drive the laser. Under certain conditions, optoelectronic feedback can drive semiconductor lasers into nonlinear oscillations, such as regular pulsing, quasi-periodic pulsing, or chaotic pulsing. The rich nonlinear dynamics of semiconductor lasers with optoelectronic feedback have been demonstrated in 


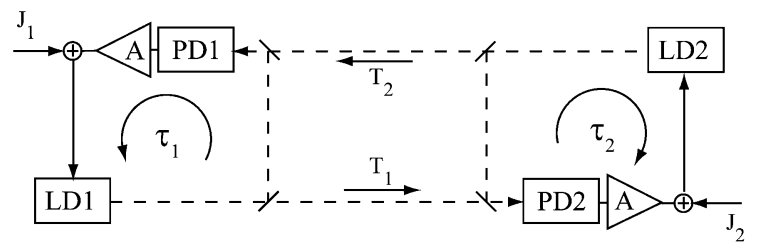

Fig. 1. Schematics of mutually coupled semiconductor lasers with optoelectronic feedback. LD: laser diode. PD: photodetector. A: amplifier. $\tau$ : feedback delay time. $T$ : mutual coupling delay time. $J$ : bias current.

[5], [6]. To study the coupling effects the two semiconductor lasers are further linked together through a mutual optoelectronic coupling. In Fig. 1, the output of LD1 is split into two parts. One part is fed back to PD1 to complete the optoelectronic feedback loop and the other part is sent to PD2 for optoelectronic coupling to LD2. Meanwhile, the output of LD2 is similarly split into two parts. One part is fed back to PD2 to complete the feedback loop, and the other part is sent to PD1 for optoelectronic coupling to LD1.

The two semiconductor lasers can be modeled by the rate equations of the intracavity photon density $S$ and the carrier density $N$

$$
\begin{aligned}
\frac{d S_{1,2}}{d t}= & -\gamma_{\mathrm{c} 1, \mathrm{c} 2} S_{1,2}+\Gamma g_{1,2}\left(N_{1,2}, S_{1,2}\right) S_{1,2} \\
& +2 \sqrt{S_{0} S_{1,2}} F_{\mathrm{s} 1, \mathrm{~s} 2} \\
\frac{d N_{1,2}}{d t}= & \frac{J_{1,2}}{e d}\left[1+\xi_{\mathrm{f} 1, \mathrm{f} 2} y_{\mathrm{f} 1, \mathrm{f} 2}\left(t-\tau_{1,2}\right)\right. \\
& \left.+\xi_{\mathrm{c} 1, \mathrm{c} 2} y_{\mathrm{c} 1, \mathrm{c} 2}\left(t-T_{2,1}\right)\right] \\
& -\gamma_{\mathrm{s} 1, \mathrm{~s} 2} N_{1,2}-g_{1,2}\left(N_{1,2}, S_{1,2}\right) S_{1,2} \\
y_{\mathrm{f} 1, \mathrm{f} 2}(t)= & \int_{-\infty}^{t} d \eta f_{1,2}(t-\eta) S_{1,2}(\eta) / S_{0} \\
y_{\mathrm{c} 1, \mathrm{c} 2}(t)= & \int_{-\infty}^{t} d \eta f_{1,2}(t-\eta) S_{2,1}(\eta) / S_{0} .
\end{aligned}
$$

The indexes 1 and 2 refer to LD1 and LD2, respectively. The signal $\xi_{\mathrm{f} 1, \mathrm{f} 2} y_{\mathrm{f} 1, \mathrm{f} 2}\left(t-\tau_{1,2}\right)$ in (2) is the feedback signal of LD1 or LD2, where $\xi_{\mathrm{f} 1, \mathrm{f} 2}$ is the feedback strength and $\tau_{1,2}$ is the feedback delay time. As is shown in (3), $y_{\mathrm{f} 1, \mathrm{f} 2}(t)$ is the convolution integral of $S_{1,2}(t)$ and the frequency response function $f_{1,2}(t)$ of the photodetector-amplifier combination in the loop of LD1, LD2. Meanwhile, the signal $\xi_{\mathrm{c} 1, \mathrm{c} 2} y_{\mathrm{c} 1, \mathrm{c} 2}\left(t-T_{2,1}\right)$ is the coupling signal from LD2 to LD1 and LD1 to LD2, respectively, where $\xi_{\mathrm{c} 1, \mathrm{c} 2}$ is the coupling strength and $T_{2,1}$ is the coupling delay time. As is shown in (4), $y_{\mathrm{c} 1, \mathrm{c} 2}(t)$ is the convolution integral of $S_{2,1}(t)$ and the frequency response function $f_{1,2}(t)$. In the configuration of Fig. 1, both the feedback and the coupling signals are bandwidth limited by the frequency response function $f_{1,2}(t)$ of the photodetector-amplifier combination in the loop of LD1, LD2. Other parameters in the rate equations are the free-running intracavity photon density $S_{0}$ when a laser is not subject to feedback, the optical gain parameter $g(N, S)$, the bias current density $J$, the cavity photon decay rate $\gamma_{c}$, the spontaneous carrier decay rate $\gamma_{\mathrm{s}}$, the confinement factor of the laser waveguide $\Gamma$, the electronic charge constant $e$, the active layer thickness $d$, and the stochastic noise term $F_{\mathrm{s}}$. For numerical purposes laser parameters are taken from [5].

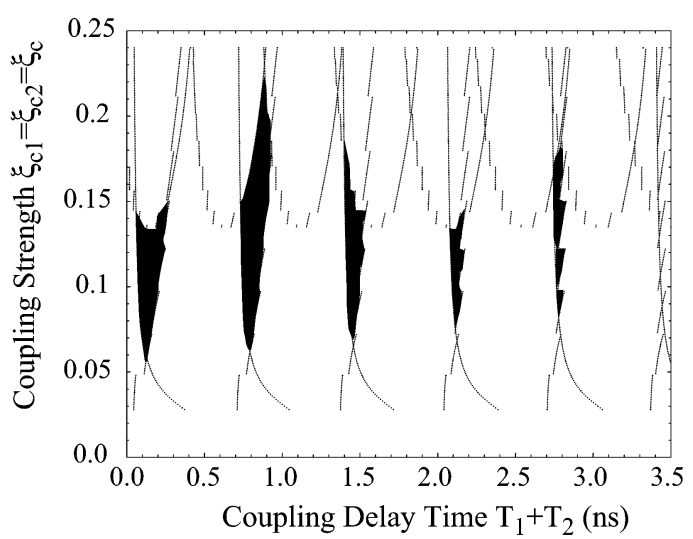

Fig. 2. Mapping of death islands in the plane of coupling strength versus total coupling delay time. The dark regions indicate death islands.

Theoretical analysis and numerical simulations have been carried out, and rich nonlinear dynamics have been observed in the system modeled by (1)-(4). (A detailed analytical and numerical study of this model will be published elsewhere [18].) In our studies, we have simplified the model by assuming an impulse response function such as $f_{1,2}(t)=\delta(t)$ because we are only interested in using the simulation results to illustrate the phenomena observed in the experiment. We have found that such simplification still maintains the qualitative characteristics of the dynamics although it may cause some quantitative differences between simulation and experiment [5], [6].

The two semiconductor lasers are first operated in a state of regular oscillation or quasi-periodic oscillation under the effect of optoelectronic feedback before the mutual coupling is applied. Once the mutual coupling is applied, dramatic effects can be observed on the original nonlinear oscillations. By tuning the parameters of $\xi_{\mathrm{c} 1}, \xi_{\mathrm{c} 2}, T_{1}$, and $T_{2}$, rich dynamics including "death by delay," generation of highly complex chaos and chaos synchronization can be observed. "Death by delay" is a phenomenon where two limit-cycle oscillators suddenly stop oscillating due to a time-delayed coupling between these oscillators. Fig. 2 shows numerical results of the mapping of death islands in the plane of coupling strength $\xi_{\mathrm{c} 1}=\xi_{\mathrm{c} 2}=\xi_{\mathrm{c}}$ versus the coupling delay time $T_{1}+T_{2}$. To our knowledge, this is the first time the "death by delay" phenomenon is reported in any semiconductor laser system. In this figure, $\tau_{1}=\tau_{2}=1.25 \mathrm{~ns}$ and $\xi_{\mathrm{f} 1}=\xi_{\mathrm{f} 2}=0.3$ are used. The dark areas are the regions for death islands. Multiple death islands are found when the total coupling delay time is increased. We will show in the following section that these results qualitatively agree with experiments. Other interesting phenomena such as generation of chaos and a quasi-periodicity route to chaos are also found in the simulation and will be reported elsewhere. In the following sections, we will focus on the experimental results about the dynamics and the evolution of dynamics of the mutually coupled semiconductor lasers.

\section{EXPERIMENTAL OBSERVATION OF DEATH BY DELAY}

"Death by delay" is a very interesting and important phenomenon where two limit-cycle oscillators suddenly stop oscillating due to a time-delayed coupling between the two oscillators. This 

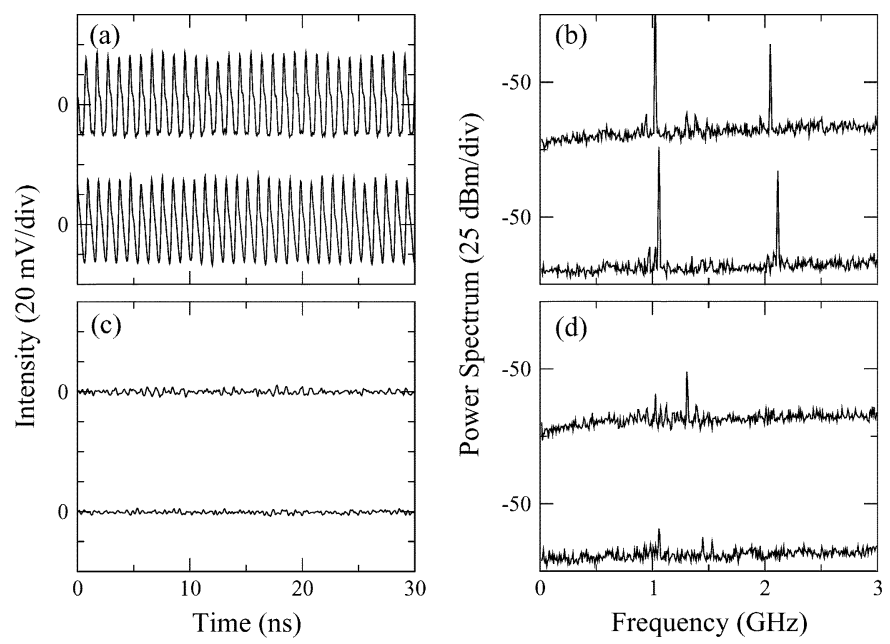

Fig. 3. Characteristics of "death by delay." (a) and (b) Time series and power spectra, respectively, of the two lasers before mutually coupling. (c) and (d) Ccorresponding plots after mutually coupling. The time series and the power spectra from PD2 are down shifted for clear comparison.

phenomenon has been theoretically predicted in coupled oscillators and has been experimentally observed in coupled circuits and biological systems [19]-[21]. Using the setup in Fig. 1, we can experimentally investigate the effect of "death by delay" in semiconductor laser oscillators.

In the experiments, the lasers are InGaAsP-InP single-mode DFB lasers both operating at $1.299-\mu \mathrm{m}$ wavelength and temperature stabilized at $21{ }^{\circ} \mathrm{C}$. The photodetectors are InGaAs photodetectors with a $6-\mathrm{GHz}$ bandwidth, and the amplifiers are Avantek SSF86 amplifiers with 0.4-3-GHz bandpass characteristics. The laser intensities measured by the photodetectors are recorded with a Tektronix TDS 694C digitizing real-time oscilloscope with a $3-\mathrm{GHz}$ bandwidth and a sampling rate up to $1 \times 10^{10}$ samples/s. Power spectra are measured with an HP E4407B RF spectrum analyzer that has a spectral range from 9 $\mathrm{kHz}$ to $26.5 \mathrm{GHz}$.

The phenomenon of "death by delay" is experimentally observed in the coupled system when each laser oscillates in a high-frequency limit cycle. Fig. 3 shows the time series and power spectra of the outputs from PD1 and PD2 before and after the mutual coupling is applied. In this case, the feedback delay times are measured to be $\tau_{1}=\tau_{2}=15.2 \mathrm{~ns}$ and the coupling delay times to be $T_{1}=14.8 \mathrm{~ns}$ and $T_{2}=15.7 \mathrm{~ns}$, respectively. Before mutual coupling, both lasers are operating in independent limit-cycle oscillations. The corresponding time series and the power spectra are shown in Fig. 3(a) and (b), respectively. It can be seen that the two lasers oscillate at regular pulsing states with almost the same fundamental frequencies of about $1 \mathrm{GHz}$. However, as soon as mutual coupling is applied, the original large-amplitude oscillations are suddenly quenched to almost zero amplitudes. The time series and the power spectra in this state are shown in Fig. 3(c) and (d), respectively. It can be seen that the oscillations have disappeared while the amplitudes of both time series are almost zero. Moreover, the flat spectra in Fig. 3(d) are close to the noise floor. The residual fluctuations in the two lasers are caused by noise from the lasers and the electronic circuits in the system. Nevertheless, comparing the time series and the power spectra before and after mutual coupling,

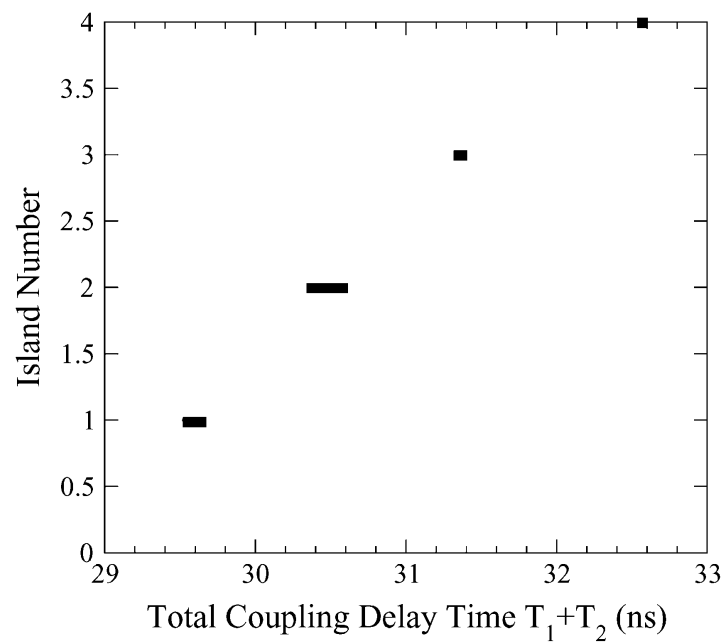

Fig. 4. Multiple death islands versus the total coupling delay time, $T_{1}+T_{2}$. The dark regions indicate death islands.

it is clear that the oscillations are almost completely quenched by the mutual coupling between the two oscillators.

The phenomenon of the death of the oscillations happens only at certain coupling delay times. As the two lasers are mutually coupled to each other through separate paths, the delay times $T_{1}$ and $T_{2}$ can be adjusted separately. We have found that it is the total mutual coupling delay time $T_{1}+T_{2}$ that determines the appearance of a death island. When $T_{1}$ is shortened or elongated around a death island, the path length corresponding to $T_{2}$ has to be adjusted reversely in order to maintain a constant $T_{1}+T_{2}$ for the two lasers to stay in the same death island. In Fig. 3, we found that $T_{1}+T_{2}=30.5 \mathrm{~ns}$ which is about twice the value of $\tau_{1}$ or $\tau_{2}$. Theoretical results in Fig. 2 have also shown the same dependence of a death island on the total mutual coupling delay time, although this comparison is only qualitative. Multiple death islands are observed when the total mutual coupling delay time $T_{1}+T_{2}$ is changed over a wide range. Fig. 4 shows four of these islands when $T_{1}+T_{2}$ is varied. When the total coupling delay time is increased, a sequence of death islands show up at multiple positions with almost equal separations. In Fig. 4, the averaged separation between the islands is about $1 \mathrm{~ns}$. This time separation matches with the inverse of the original oscillation frequency before coupling, which is about $1 \mathrm{GHz}$ as shown in Fig. 3(b). As limited by the physical length of the setup and the phase delay caused by the electronic components, the minimum achievable mutual coupling delay time, $T_{1}+T_{2}$, is about $29.5 \mathrm{~ns}$ in our experiment. The value of $T_{1}+T_{2}$ can be further varied over a range of about $4 \mathrm{~ns}$. In reality, there is always a bandwidth limitation from the components such as the amplifiers, the photodetectors, and even the lasers. Consequently, the mutually coupled semiconductor laser system is not only highly nonlinear but also highly dispersive. It has been found that such bandwidth limitation can cause quantitative, though not qualitative, differences in characteristics, compared with a simplified simulation model without bandwidth limitation [5], [6]. Therefore, the experimental results in Fig. 4 should only be qualitatively compared with the theoretical results in Fig. 2. In both figures, we can see that the death islands repeat with equal separation relating to the oscillation frequency. In semiconductor 

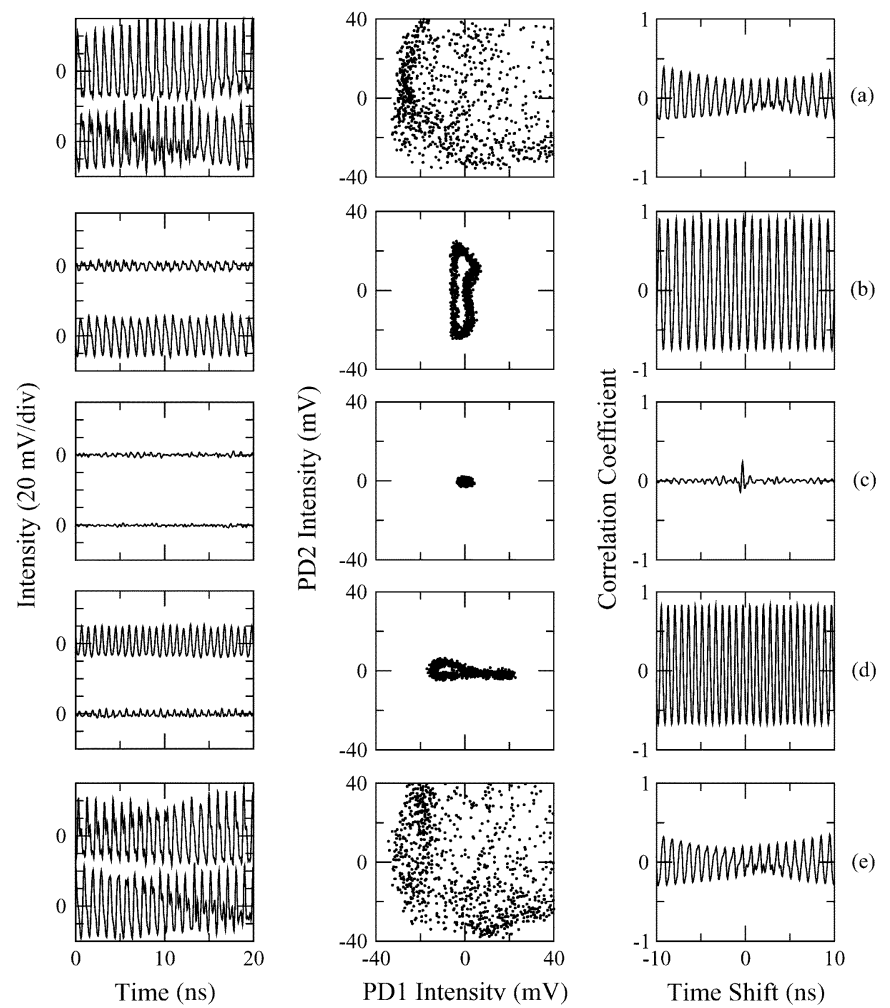

Fig. 5. Sequence of dynamical states showing the evolution of the laser characteristics into and out of a death island. The first column shows the time series; the second column shows the correlation plot of output from PD1 versus that from PD2; and the third column shows the shifted correlation coefficient between the outputs from PD1 and PD2.

lasers with optoelectronic feedback the limit-cycle oscillation is generated by the delayed feedback. A certain oscillation frequency is usually generated at multiple feedback delay times which have a separation of about the period of the oscillation frequency [5]. Therefore, in our system we have seen that the death islands appear at certain total coupling delay times which have a specific relation with the feedback delay time. Nevertheless, the phenomenon of "death by delay" has been observed in many other mutually coupled limit-cycle oscillators [19]-[21] which do not necessarily have a delayed feedback. This phenomenon is general and does not depend on how the limit-cycle oscillations are generated.

To show how the lasers develop into and out of a death island, the outputs from PD1 and PD2 are recorded sequentially as the total coupling delay time is increased. Fig. 5 illustrates the different dynamical states that are obtained. The feedback delay times are fixed at $\tau_{1}=\tau_{2}=15.2 \mathrm{~ns}$. From top to bottom, $T_{1}+T_{2}$ is $29.9,30.3,30.5,30.7$, and $30.9 \mathrm{~ns}$, respectively. Each row represents data for one dynamical state. The three columns represent the changes in time series, correlation plot, and shifted correlation coefficient [22], respectively. The time series show the intensity fluctuations of the outputs from PD1 and PD2. The correlation plot is obtained by plotting the output from PD1 versus that from PD2. The shifted correlation coefficient is calculated between the two outputs when the waveform from PD2 is time shifted gradually. In the first state shown in Fig. 5(a), the outputs from the two lasers exhibit large-amplitude oscillations and the two outputs are only slightly correlated. In the correla- tion plot, the data points scatter over a wide area, which confirms the low correlation between the two outputs. The maximum of the shifted correlation coefficient is less than 0.5 over the entire range of the relative time shift. In the second state shown in Fig. 5(b), the coupling delay time is increased, resulting in a dramatic decrease in the oscillation from PD1. Nevertheless, the outputs become highly correlated because in this situation, only LD2 generates an oscillation and LD1 is modulated by this oscillation through the mutual coupling. The correlation plot shows a simple pattern which indicates that the two outputs are correlated with a phase shift. In the shifted correlation coefficient, there are regularly oscillating correlation peaks, which mean that the two waveforms are periodically correlated. The correlation coefficient exhibits maxima each time the waveform from PD2 is phase shifted by $2 \pi$. As the coupling delay time increases further, the amplitude of the oscillating LD2 also shrinks to almost zero amplitude as the two coupled lasers enter a death island. Such a death state is shown in Fig. 5(c). The time series show no oscillations in both lasers. The correlation plot shrinks to a very small spot, indicating the onset of a death state. There are no significant correlation peaks because both lasers are in a death state. The small peak at the center is caused by residual fluctuations of the outputs of both lasers. After passing the death island, the oscillation amplitude of LD1 starts to increase significantly. LD2 has no dynamical oscillation by itself but is weakly modulated by the coupled signal from LD1. This situation is shown in Fig. 5(d). The time series, correlation plot, and shifted correlation coefficient are similar to the corresponding plots in row (b), except that the roles of the two lasers are now interchanged. By increasing the coupling delay time further, the two lasers start oscillating with large amplitudes again, and similar plots as in Fig. 5(a) are observed in the last state as is shown in row Fig. 5(e). The whole sequence repeats again as the coupling delay time continues to increase through the next death island.

To study the mechanism that yields to the "death by delay" phenomenon, the configuration in Fig. 1 is slightly modified. First, the feedback signals in both lasers are blocked and only mutual coupling remains. Under this situation no oscillation is observed because the mutual coupling strength is weak under this operating condition. Next, the feedback loop in LD1 is unblocked while the feedback loop in LD2 is still blocked. Therefore, only LD1 oscillates before mutual coupling. With mutual coupling, it is observed that the oscillation in LD1 is also coupled to LD2. This dynamical state is very similar to that shown in Fig. 5(d). Since the coupling strength is weak, this coupling of oscillation is caused by the mechanism of weak modulation. The weakly modulated signal from LD2 is further coupled back to LD1 again. In this way, the total mutual coupling loop, including LD2 as a signal relay, forms a second feedback loop for the oscillating LD1. When the phase of the electronic signal that is fed back through this second loop is in antiphase $\left(180^{\circ}\right.$ shifted from the original oscillation), the second loop creates a negative feedback that can quench the original oscillation of LD1. In this case, the same parameters as those in Fig. 4 are used whenever they are applicable. Fig. 6(a) shows the death islands caused by mutual coupling when only LD1 has a feedback loop but LD2 does not. Similarly, Fig. 6(b) shows the death islands when LD2 has its own feedback loop but LD1 does not. As 

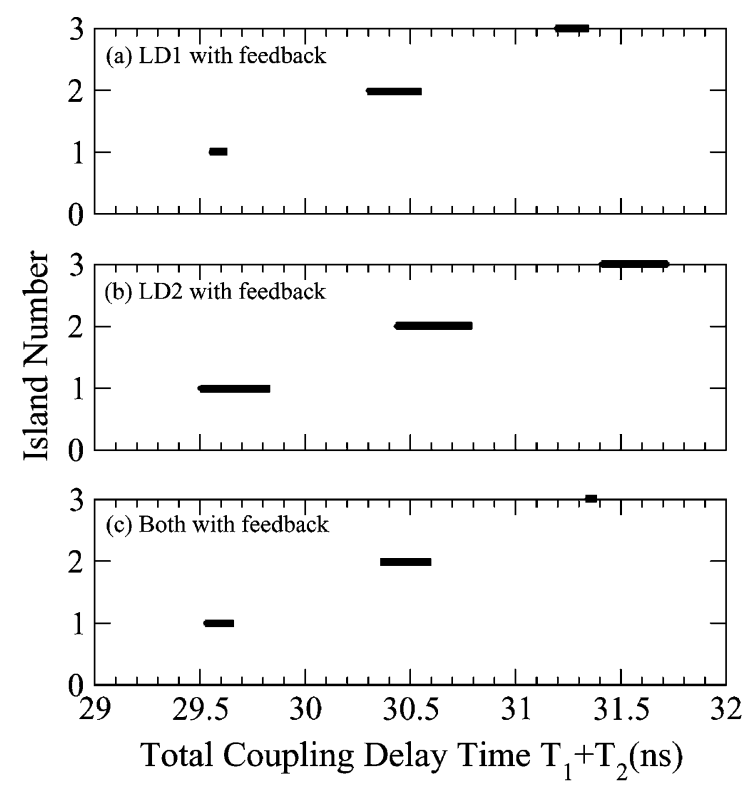

Fig. 6. Multiple death islands versus the total coupling delay time, $T_{1}+T_{2}$, obtained with different configurations. (a) Only LD1 has optoelectronic feedback. (b) Only LD2 has optoelectronic feedback. (c) Both LD1 and LD2 have optoelectronic feedback. In all three cases, LD1 and LD2 are mutually coupled together. The dark regions indicate death islands.

can be seen the death islands in Fig. 6(a) and (b) are very much lined up at the same total mutual coupling delay times. This is because the two lasers oscillate at almost the same frequency when they have feedback. The slight shifts in the positions of the islands in Fig. 6(a) and (b) are caused by the slight difference in the two oscillation frequencies due to small parameter mismatch. Fig. 6(c) also shows the same death islands as in Fig. 4 when both lasers are subject to feedback. Comparing Fig. 6(c) with Fig. 6(a) and (b), it is clear that the islands in Fig. 6(c) exist only when death islands show up in both Fig. 6(a) and (b). In the three cases shown in Fig. 6(a)-(c), the mutual coupling serves as negative feedback to both lasers, thereby inducing oscillation quenching.

Based on our results, we can thus see that the oscillation quenching seen in the "death by delay" phenomenon is caused by a negative feedback loop introduced by mutual coupling. The dependence of the death islands on the total mutual coupling delay time confirms our conclusion because the total mutual coupling delay time is exactly the delay time in this negative feedback loop created by the mutual coupling. Since phase shifts repeat after each period of oscillation, the death islands can be expected to repeat when the total mutual coupling delay time is changed by a period of oscillation. This is in agreement with our results from Figs. 4 and 6. When mutual coupling is introduced, it is clear that both lasers experience the same total mutual coupling delay time since the path length the signals traverse is the same. Therefore, in order to achieve "death by delay" in both of the laser oscillators, the original oscillating frequencies in the oscillators have to be matched.

\section{BIFURCATION TO CHAOS}

In the experiment discussed in the preceeding section, two bandpass filters are inserted into the setup after the two am-
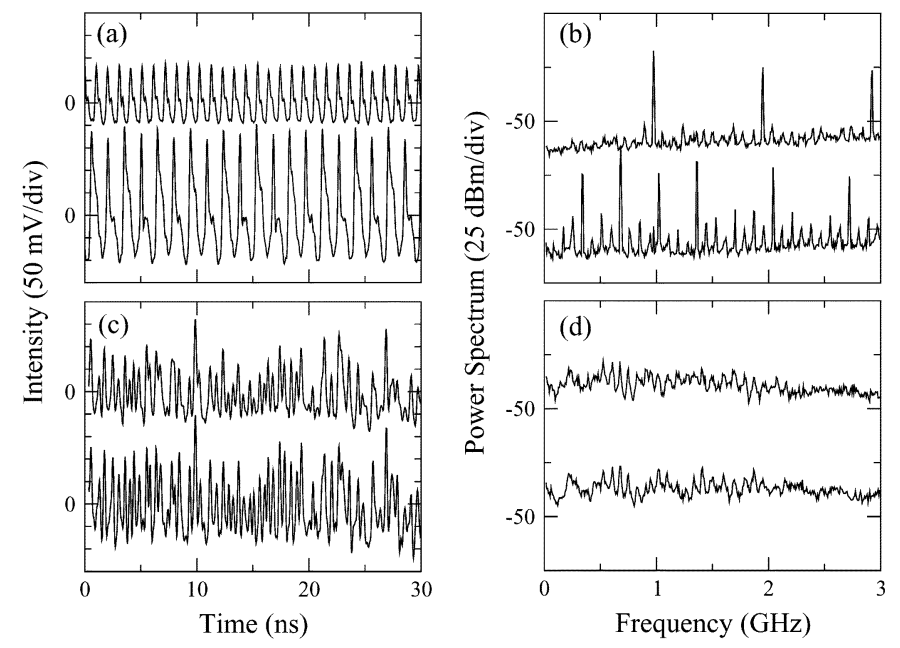

Fig. 7. Characteristics of chaos induced by mutual coupling. (a) and (b) Time series and power spectra, respectively, of the two lasers before mutually coupling. (c) and (d) Corresponding plots after mutually coupling.

plifiers in order to ensure the same oscillation frequencies in both lasers before coupling. Under this situation, the oscillation frequencies are confined within the passband of the filters. The purpose of this section is to study the chaotic states of mutually coupled oscillators. Since the onset of a chaotic state is accomplished by a broad spectrum, the bandpass filters are removed to increase the response bandwidths of the two oscillators. Before mutual coupling, the two lasers are driven into some simple pulsing states such as regular pulsing or quasi-periodic pulsing states. The time series and power spectra of the outputs from PD1 and PD2 before coupling are shown in Fig. 7(a) and (b), respectively. In this case, the feedback delay times are measured to be $\tau_{1}=\tau_{2}=15.4 \mathrm{~ns}$. As is shown, the waveform from PD1 is a regular pulsing state with one fundamental frequency and its harmonics while that from PD2 is a quasi-periodic pulsing state with two incommensurate fundamental frequencies. The difference in the two waveforms are caused by the slight differences in the intrinsic laser parameters and operating conditions. With mutual coupling, highly complex chaotic outputs from the two lasers are observed. In this case, the mutual coupling strength is much stronger than that of the preceeding section. Fig. 7(c) and (d), respectively, show the time series and power spectra of the outputs from PD1 and PD2 when chaos is generated in both lasers due to the mutual coupling. The coupling delay times are also measured to be $T_{1}=T_{2}=15.4 \mathrm{~ns}$. The chaotic waveforms have dramatic fluctuations in the time series and very broad distributions in the power spectra, which indicate that the mutual coupling can destabilize two oscillators into highly chaotic states.

The characteristics of a typical quasi-periodic route to chaos are shown in Fig. 8. The feedback delay times are still $\tau_{1}=\tau_{2}=$ $15.4 \mathrm{~ns}$. The figure shows a sequence of three dynamical states which are regular pulsing (RP), two-frequency quasi-periodic pulsing $(\mathrm{Q} 2)$, and chaotic pulsing $(\mathrm{C})$, respectively, obtained by changing $T_{2}$ from 15.4 to $15.3 \mathrm{~ns}$ first and then slightly reduce the coupling strength $\xi_{\mathrm{c} 2}$ in the last state. The parameter $T_{1}$ is fixed at $15.4 \mathrm{~ns}$ in the three states. This example sequence is obtained with the configuration where the two lasers are mutually 

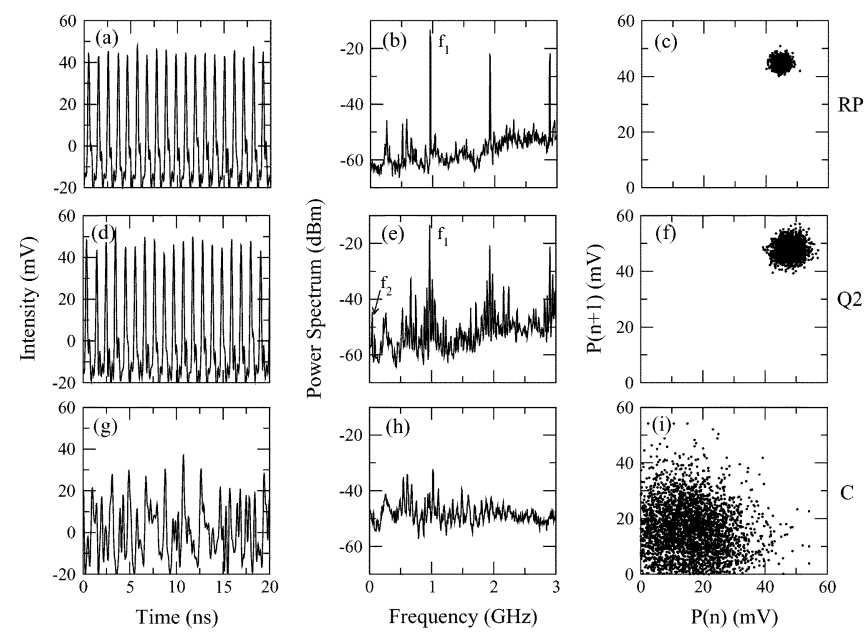

Fig. 8. Quasi-periodic pulsing route to chaos. RP: regular pulsing state. Q2: two-frequency quasi-periodic pulsing state. C: chaotic pulsing state. The first column shows the time series; the second column shows the power spectra; and the third column shows the phase portraits.

coupled while having optoelectronic feedback on both of them. For each dynamical state, the time series, power spectrum, and phase portrait from the system output of PD2 are plotted in the first, second, and third columns, respectively. The output of the system from PD1 is similar to that from PD2 for each dynamical state.

In Fig. 8(a)-(c), the system is in a regular pulsing state. The time series shows a train of regular pulses with almost constant maxima of the pulsing intensity and time interval. Since the pulses are very short and the digitization speed of the oscilloscope is limited to $1 \times 10^{10} \mathrm{Samples} / \mathrm{s}$, there are digitization errors especially at the peak of the pulses. Therefore, the fluctuations in the peak intensities of the pulses are mainly caused by digitization errors. Nevertheless, the corresponding power spectrum has only one fundamental pulsing frequency, $f_{1}$, which is about $1 \mathrm{GHz}$, and its harmonics. There are some low frequency spikes which are due to resonance oscillation from the amplifiers. The phase portrait is obtained by recording a peak sequence $\mathrm{P}(\mathrm{n})$ at the local intensity maxima of a pulse train and further plotting $\mathrm{P}(\mathrm{n})$ versus $\mathrm{P}(\mathrm{n}+1)$. In the regular pulsing state, the output has a constant peak intensity, and the phase portrait shows only one spot. The fluctuations in the time series and, correspondingly, the scattering in the phase portrait are mainly caused by the noise in the system and the digitization errors from the oscilloscope. When $T_{2}$ is decreased, the system enters into a two-frequency quasi-periodic pulsing state with the pulsing intensity modulated at a certain frequency $f_{2}$ as shown in Fig. 8(d)-(f). The time series shows the characteristic irregular modulation of quasi-periodic pulsing on the peak intensity although there are still a lot of digitization errors. Nevertheless, the characteristics of quasi-periodicity is clear in the power spectrum. Besides the pulsing frequency $f_{1}$, an incommensurate frequency $f_{2}$ indicating the modulation of the peak intensity shows up at low frequency. This $f_{2}$ is related to the total coupling delay time $T_{1}+T_{2}$ of the mutual coupling loop because of the nonlinear interaction in this system. Both $f_{1}$ and $f_{2}$ are indicated in the figure for clarity. The other spectrum spikes are mainly the harmonics and combinations of $f_{1}$ and $f_{2}$. The
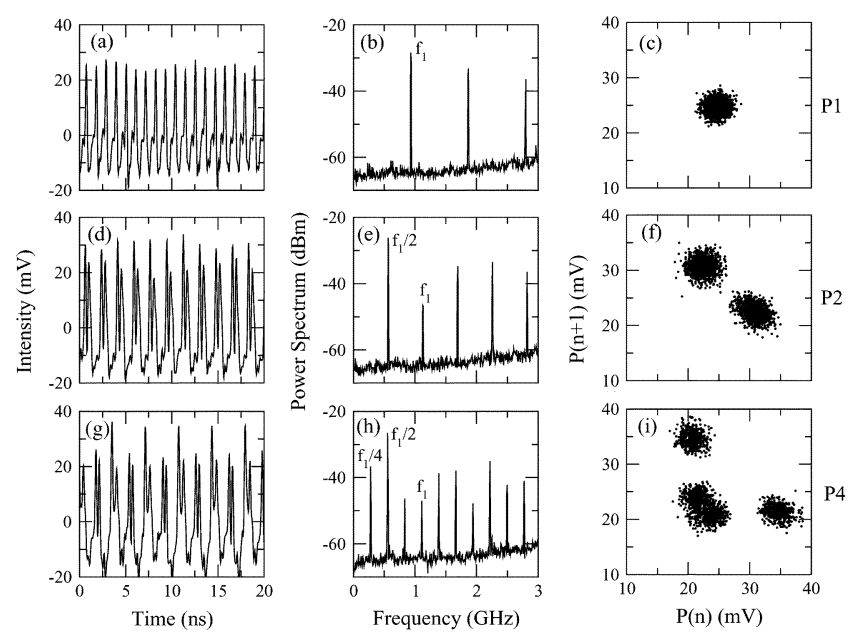

Fig. 9. Characteristics of period-doubling bifurcation. P1: period-one pulsing state. P2: period-two pulsing state. P4: period-four pulsing state. The first column shows the time series; the second column shows the power spectra; and the third column shows the phase portraits.

appearance of two incommensurate frequencies, $f_{1}$ and $f_{2}$, indicates quasi-periodicity. In the phase portrait, the data points are still scattered due to noise and digitization errors. However, we can see that the distribution in Fig. 8(f) is more scattered than that in Fig. 8(c) due to the modulation on the maxima of the pulse intensity. In Fig. 8(g)-(i), when $\xi_{c 2}$ is now decreased, the system enters into a chaotic pulsing state. From the time series, we find that both the pulse intensity and the pulsing interval vary chaotically. At the same time, the power spectrum is broadened indicating the onset of chaos. The phase portrait shows a highly scattered distribution in a large area. The system is thus shown to enter a chaotic pulsing state through a quasi-periodic route. Our numerical simulation also shows a quasi-periodicity route to chaos which qualitatively matches with our experiments. Such simulation results will be reported elsewhere.

While Fig. 8 shows the characteristics of a typical quasi-periodic bifurcation to chaos, Fig. 9 shows the characteristics of a typical period-doubling bifurcation. Here, as an example, we use a sequence obtained with a configuration where the two lasers are mutually coupled but only LD1 has optoelectronic feedback. Each column has the same meaning as that in Fig. 8. The feedback delay time is $\tau_{1}=13.7 \mathrm{~ns}$ and the coupling delay time is $T_{1}=16.2 \mathrm{~ns}$. From top to bottom, $T_{2}$ is $15.4,15.8$, and $16.4 \mathrm{~ns}$, respectively. The first row shows a period-one (P1) pulsing state. The pulse train has a constant peak intensity and the power spectrum has only one fundamental frequency, $f_{1}$. In the phase portrait, there is only one single spot which corresponds to the signal peak intensity in the pulse train. The second row shows a period-two (P2) pulsing state, where the pulse intensity has two distinctive values which repeat one after the other. In the power spectrum, except the pulsing frequency $f_{1}$, the subharmonic $f_{1} / 2$ also shows up. The other frequency peaks are the harmonics and combinations of $f_{1}$ and $f_{1} / 2$. In the phase portrait, the two scattered spots that are clearly seen are characteristic of a period-two state. The third row shows a period-four (P4) pulsing state. Now, there are four distinctive values in the pulse intensity. In the power spectrum, frequency components at $f_{1} / 4, f_{1} / 2, f_{1}$, and their combinations are all observed. Four 

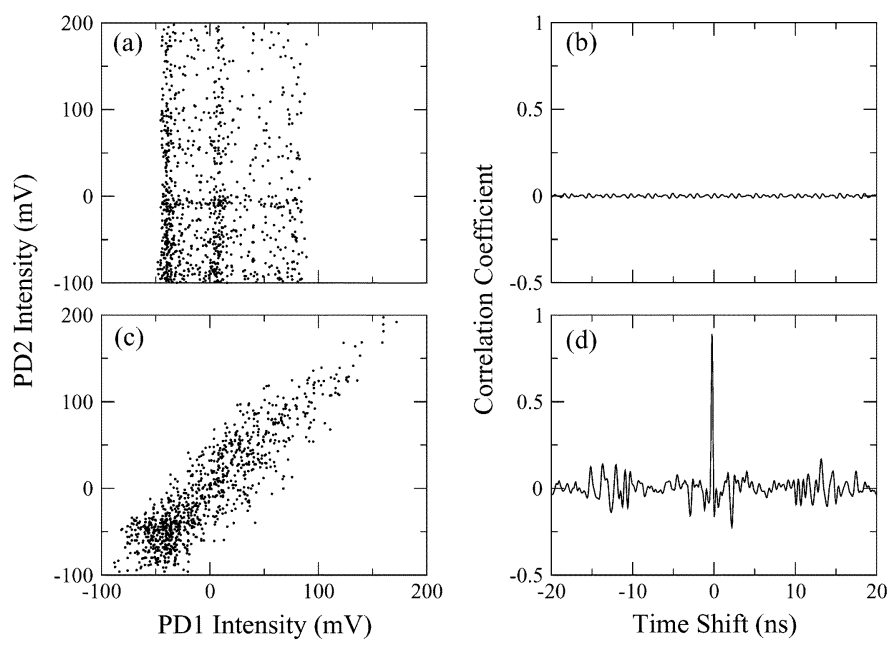

Fig. 10. Chaos synchronization induced by mutual coupling. (a) and (b) Correlation plot and shifted correlation coefficient, respectively, of the outputs from PD1 and PD2 before mutually coupling. (c) and (d) Corresponding plots after mutually coupling.

distinctive spots are found in the phase portrait with two of them located close to each other. Within the evolution of the above three dynamical states, no intermediate quasi-periodic state is observed. Therefore, it is shown that a period-doubling bifurcation from P1, P2, to P4 is observed in the mutually coupled semiconductor lasers. The period-doubling bifurcation can also lead the system into a chaotic pulsing state that is similar to the one shown in Fig. 8 but is not shown in Fig. 9 again. Thus, the system can have a quasi-periodic pulsing route, a period-doubling pulsing route, or a mixture of these two bifurcations to chaos. Since this system is highly nonlinear and dispersive as we have discussed previously, it is not surprising that there are two bifurcation routes to chaos. Furthermore, this highly nonlinear and dispersive characteristics can also cause the dynamics to change the temporal and spectral structures when the operating parameters are changed.

\section{ChaOs SynChronization}

It is shown in Section IV that chaotic states can be generated in two mutually coupled semiconductor lasers through different routes. When such chaotic states are generated in the two lasers simultaneously, it is of great interest and importance to study the correlation between the two chaotic outputs. In this system, the mutual coupling not only drives the two lasers into chaos but can also synchronize such chaos. The time series and power spectra of the system outputs from PD1 and PD2 before and after mutual coupling have been shown in Fig. 7. Before mutual coupling, the two outputs are independent oscillations with no correlation, as is seen in Fig. 7(a) and (b). After mutual coupling, the two waveforms become highly correlated while the power spectra are very similar to each other, as can be seen in Fig. 7(c) and (d). In Fig. 10, we show the correlation plot and the shifted correlation coefficient between the outputs before and after mutual coupling. The coupling and feedback delay times are the same as those in Fig. 7. Fig. 10(a) and (b) show the characteristics before mutual coupling when the two lasers oscillate in some simple pulsing states. Even though the pulsing states are simple, the waveforms are completely uncorrelated. The correlation plot shows very scattered distribution, and the shifted correlation coefficient is practically zero everywhere. However, as soon as mutual coupling is applied, the outputs of the two lasers dramatically change into highly chaotic oscillations. Both lasers are then in chaotic states, indicating that the mutual coupling has destabilized the coupled oscillators. Although the outputs from the two lasers are now very complex chaotic waveforms, they are in fact highly correlated. The correlation plot shows a nice distribution along the $45^{\circ}$ diagonal line in Fig. 10(c) and the shifted correlation coefficient shows a very sharp peak in Fig. 10(d). Both indicate a high quality of chaos synchronization between the two waveforms. The observed correlation coefficient is as high as 0.9 and there are no other correlation peaks except the central one. The position of the correlation peak indicates the time shift between the two chaotic waveforms. In Fig. 10(d), this correlation peak is found to be at zero time shift which means that the two waveforms are synchronized in time. The identical synchronization is achieved because we have $\tau_{1}=\tau_{2}=T_{1}=T_{2}=15.4 \mathrm{~ns}$ and a close match of all the other parameters between the two lasers. Furthermore, we found that the synchronization is maintained when $T_{1}+T_{2}$ is kept a constant but the specific values of $T_{1}$ and $T_{2}$ are changed, respectively. In such a case, the correlation peak shifts away from the center by the amount of $\left(T_{1}-T_{2}\right) / 2$, indicating a time shifted synchronization between the chaotic waveforms.

\section{CONCLUSION}

Mutually coupled semiconductor lasers are investigated in order to study the effect of mutual coupling on semiconductor lasers in particular and such effect on mutually coupled dynamical systems in general. It is found that the time delay of coupling plays an important role on the dynamics and synchronization in the mutually coupled semiconductor lasers. Depending on the coupling delay time and the coupling strength, mutual coupling can act as a negative feedback to stabilize the coupled oscillators, or it can increase the complexity of the system to drive it into highly complex chaos. A route to chaos of quasi-periodicity, period-doubling, or a combination of both, is found in such systems. Although the chaotic waveforms are highly complex with very broad spectra, high quality of synchronization between the chaotic waveforms is found. Such synchronization is achieved because of the effect of mutual coupling and the symmetric arrangement between the two lasers. From these demonstrations, it is shown that mutual coupling plays very important roles in coupled dynamical systems.

\section{REFERENCES}

[1] H. Olesen, J. H. Osmundsen, and B. Tromborg, "Nonlinear dynamics and spectral behavior for an external cavity laser," IEEE J. Quantum Electron., vol. QE-22, pp. 762-773, June 1986.

[2] G. Giacomelli, M. Calzavara, and F. T. Arecchi, "Instabilities in a semiconductor laser with delayed optoelectronic feedback," Opt. Comm., vol. 74, pp. 97-101, Dec. 1989.

[3] V. Ahlers, U. Parlitz, and W. Lauterborn, "Hyperchaotic dynamics and synchronization of external-cavity semiconductor lasers," Phys. Rev. E, vol. 58, no. 6, pp. 7208-7213, Dec. 1998.

[4] J. Ohtsubo, "Feedback induced instability and chaos in semiconductor lasers and their applications," Opt. Rev., vol. 6, pp. 1-15, Jan.-Feb. 1999. 
[5] S. Tang and J. M. Liu, "Chaotic pulsing and quasi-periodic route to chaos in a semiconductor laser with delayed optoelectronic feedback," IEEE J. Quantum Electron., vol. 37, no. 3, pp. 329-336, Mar. 2001.

[6] F. Y. Lin and J. M. Liu, "Nonlinear dynamics of a semiconductor laser with delayed negative optoelectronic feedback," IEEE J. Quantum Electron., vol. 39, pp. 562-568, Apr. 2003.

[7] H. F. Chen and J. M. Liu, "Open-loop chaotic synchronization of injection-locked semiconductor lasers with gigahertz range modulation," IEEE J. Quantum Electron., vol. 36, pp. 27-34, Jan. 2000.

[8] H. Fujino and J. Ohtsubo, "Experimental synchronization of chaotic oscillations in external-cavity semiconductor lasers," Opt. Lett., vol. 25, no. 9, pp. 625-627, May 2000.

[9] Y. Liu, P. Davis, and T. Aida, "Synchronized chaotic mode hopping in DBR lasers with delayed optoelectronic feedback," IEEE J. Quantum Electron., vol. 37, pp. 337-352, Mar. 2001

[10] S. Tang and J. M. Liu, "Synchronization of high-frequency chaotic optical pulses," Opt. Lett., vol. 26, no. 9, pp. 596-598, May 2001.

[11] "Special issue on applications of chaos in modern communication systems," IEEE Trans. Circuits Syst. I, vol. 48, Dec. 2001

[12] "Feature section on optical chaos and application to cryptography," IEEE J. Quantum Electron., vol. 38, Sept. 2002.

[13] R. Vicente, T. Perez, and C. Mirasso, "Open versus closed loop performance of synchronized chaotic external-cavity semiconductor lasers," IEEE J. Quantum Electron., vol. 38, pp. 1197-1204, Sept. 2002.

[14] S. Tang and J. M. Liu, "Message encoding-decoding at 2.5 Gbits/s through synchronization of chaotic pulsing semiconductor lasers," Opt. Lett., vol. 26, pp. 1843-1845, Dec. 2001.

[15] A. Hohl, A. Gavrielides, T. Erneux, and V. Kovanis, "Localized synchronization in two coupled nonidentical semiconductor lasers," Phys. Rev. Lett., vol. 78, no. 25, pp. 4745-4748, June 1997.

[16] T. Heil, I. Fischer, W. Elsasser, J. Mulet, and C. R. Mirasso, "Chaos synchronization and spontaneous symmetry-breaking in symmetrically delay-coupled semiconductor lasers," Phys. Rev. Lett., vol. 86, pp. 795-798, Jan. 2001.

[17] J. Mulet, C. Masoller, and C. R. Mirasso, "Modeling bidirectionally coupled single-mode semiconductor lasers," Phys. Rev. A, vol. 65, no. 063815, June 2002

[18] R. Vicente, S. Tang, J. Mulet, C. R. Mirasso, and J. M. Liu, "Synchronization properties of two self-oscillating semiconductor lasers subject to delayed optoelectronic mutual-coupling", submitted for publication.

[19] D. V. R. Reddy, A. Sen, and G. L. Johnston, "Experimental evidence of time-delay-induced death in coupled limit-cycle oscillators," Phys. Rev. Lett., vol. 85, pp. 3381-3384, Oct. 2000.

[20] A. Takamatsu, T. Fujii, and I. Endo, "Time delay effect in a living coupled oscillator system with the plasmodium of Physarum polycephalum," Phys. Rev. Lett., vol. 85, pp. 2026-2029, Aug. 2000.

[21] R. Herrero, M. Figueras, J. Rius, F. Pi, and G. Orriols, "Experimental observation of the amplitude death effect in two coupled nonlinear oscillators," Phys. Rev. Lett., vol. 84, pp. 5312-5315, June 2000.

[22] S. Tang and J. M. Liu, "Chaos synchronization in semiconductor lasers with optoelectronic feedback," IEEE J. Quantum Electron., vol. 39, pp. 708-715, June 2003.

Shuo Tang received the B.S. and M.S. degrees in electronics from Peking University, Beijing, China, in 1992 and 1997, respectively, and the Ph.D. degree in electrical engineering from the University of California, Los Angeles, in 2003.

Her research interests include optical communication systems, semiconductor lasers, nonlinear dynamics, and biological imaging.
Raúl Vicente was born in Palma de Mallorca, Spain, in 1979. He received the degree in physics (Extraordinary Award) from the Universitat de les Illes Balears, Palma de Mallorca, in 2001, where he is currently working toward the Ph.D. degree in laser physics.

His research interests include semiconductor laser dynamics, nonlinear dynamics, and cryptography.

Margaret C. Chiang received the B.S. degree in electrical engineering from the University of California, Los Angeles, in 2002, where she is currently working toward the Ph.D. degree in electrical engineering.

Her research interests include semiconductor lasers and nonlinear dynamics, with an emphasis on biomedical applications.

Claudio R. Mirasso was born in Buenos Aires, Argentina, in 1960. He received the M.Sc. and Ph.D. degrees in physics from the Universidad Nacional de $\mathrm{La}$ Plata, Buenos Aires, in 1984 and 1989, respectively.

From 1990 to 1992, he was Postdoctoral Researcher with the Physics Department, Universitat de les Illes Balears, Palma de Mallorca, Spain. From 1992 to 1994, he was a Visiting Professor with the Modern Physics Department, Universidad de Cantabria, Cantabria, Spain, and the Physics Department, Universitat de les Illes Balears, respectively. Between 1994 and 1995, he was a Scientific Researcher with the Consejo Superior de Investigaciones Científicas, Madrid, and a European Postdoctoral Researcher with the Physics Department, the Free University, Amsterdam, The Netherlands. Since 1996, he has been an Associate Professor with the Physics Department, Universitat de les Illes Balears. $\mathrm{He}$ is currently the Coordinator of the European Project OCCULT (optical chaos communication using laser-diode transmitters). He has authored or coauthored over 100 publications including about 70 journal papers. His research interests include instabilities in semiconductor lasers, synchronization, and control of chaotic semiconductor lasers, vertical cavity semiconductor lasers, and applications of nonlinear dynamics.

Jia-Ming Liu received the B.S. degree in electrophysics from National Chiao Tung University, Hsinchu, Taiwan, in 1975, and the S.M. and Ph.D. degrees in applied physics from Harvard University, Cambridge, MA, 1979 and 1982, respectively.

He was an Assistant Professor with the Department of Electrical and Computer Engineering, State University of New York at Buffalo, from 1982 to 1983 and was a Senior Member of the Technical Staff with GTE Laboratories, Inc., from 1983 to 1986 . He is currently a Professor of electrical engineering at University of California, Los Angeles. His research interests include development and application of ultrafast wavelength-tunable laser pulses, nonlinear and ultrafast processes in materials and devices, optical wave propagation, optical communications, nonlinear dynamics of lasers, and chaotic communications.

Dr. Liu became a Licensed Professional Electrical Engineer in 1977. He is a Fellow of the Optical Society of America and the American Physical Society, a Senior Member of the IEEE Laser and Electro-Optics Society, and a Founding Member of the Photonics Society of Chinese-Americans. 